\title{
Imagery effects and semantic similarity in sentence recognition memory
}

\author{
JON M. SLACK \\ Open University, Milton Keynes, England
}

\begin{abstract}
The idea that subjects often use imagery to discriminate semantically similar sentences was tested in three experiments. In the first experiment, subjects heard subject-verb-object sentences in the context of either a comprehension task or an image-generation task. Their memory for the sentences was tested using a two-alternative forced-choice recognition test in which different types of distractor sentence were used. A sentence semantically similar to the target sentence was one type; a sentence with the same subject and object nouns as the target sentence, but dissimilar in meaning, was another type; and a sentence similar in meaning to one of the stimulus sentences, but not to the target sentence, was a third type. The results showed that the image-generation instructions enhanced later recognition performance, but only for semantically similar test items. A second experiment showed that this finding only holds for highimagery sentences containing concrete noun concepts. A third experiment demonstrated that the enhanced recognition performance could not be accounted for in terms of a semantic model of test-item discrimination. Collectively, the results were interpreted as providing evidence for the notion that subjects discriminate the semantically similar test items by elaborating the sentence encodings through image processing.
\end{abstract}

The functional role of imagery within cognition remains unclear even though a large corpus of evidence implicating it as a factor in a range of cognitive tasks has accumulated (Kosslyn, 1980; Paivio, 1971). Part of this evidence suggests that imagery is used in understanding certain types of sentences (Begg \& Paivio, 1969; Glass, Eddy, \& Schwanenflugel, 1980). The present study attempted to show that imagery may play a role in the discrimination of semantically similar sentences, and demonstrated the influence of this process on recognition memory.

If subjects are asked to describe the differences in meaning between a pair of semantically close sentences, such as (S1) "The man strolled through the park," and (S2) "The man sauntered through the park," they often report using imagery to achieve the task. Their introspections suggest that they derive their responses from some form of image-comparison process. That is, imagery is used to elaborate the internal representations of the two sentences in order to maximize the semantic differences between them. Anderson and Bower (1973) argued that when a subject forms an image of a sentence, he "unpacks" its conceptual components into more primitive terms. This semantic elaboration is transformed, through various processes, into additional propositions that are connected to human associative memory's (HAM's) conceptual-propositional representation for the sentence.

If this notion of semantic elaboration through imagery is valid, then it has implications for a range of cognitive

The author's mailing address is: Department of Psychology, Open University, Walton Hall, Milton Keynes MK 7 6AA, England. tasks that have been shown to be influenced by the variable of semantic similarity. In particular, on a forced-choice recognition memory task using semantically similar test sentences, subjects will use imagery to create discriminable representations for the test items, and match these against the memory traces of the studied sentences. However, Anderson (Note 1) showed that such test items are likely to be confused, implying that subjects are unable to utilize the differentiated testtrial encodings to improve their recognition accuracy. But recognition performance is also influenced by the encoding instructions. According to the encoding specificity principle (e.g., Tulving \& Thompson, 1973), recognition performance is a function of the overlap between the encoding and retrieval environments. Thus, if subjects use imagery to produce differentiated testitem representations, these codes will be effective in augmenting recognition performance only if they produce a more sensitive match with the study-trial encodings. To maximize the overlap between the encoding and retrieval environments, it is necessary for subjects to generate images for the sentences on the study trials (Fisher \& Craik, 1980). Since Anderson's (Note 1) subjects were not instructed to produce images for the study sentences, the overlap in content between the study- and test-trial encodings was insufficient for the target and distractor sentences to be accurately discriminated.

A number of studies have shown that recognition performance is inversely related to the similarity between the old and new test items (Bower \& Glass, 1976; Nagae, 1980; Weaver \& Stanny, 1978). However, Tulving 
(1981) recently showed that the effects of similarity on recognition memory are more complex than these studies suggested. On a forced-choice recognition memory task employing pictures as stimulus items, he found that, under certain conditions, subjects are more accurate at discriminating between similar than between dissimilar test items. To explain this result, he distinguished between two types of similarity relations, perceptual and ecphoric, which independently affect recognition mem. ory performance. Perceptual similarity exists between items that are physically present at the same time, such as between alternative recognition test items on a forced-choice task, whereas ecphoric similarity is the similarity existing between a test item and information stored in memory, such as the traces of the studytrial items. Tulving's results showed that subjects were more accurate at discriminating between perceptually similar test items than between items with a low degree of perceptual similarity but with the distractor item having a high degree of ecphoric similarity. This latter similarity relation was varied by making the distractor perceptually similar to one of the study-trial pictures, but not to the target with which it was paired on the test trial. He concluded that it is necessary to isolate the effects of both types of similarity relation in discussing the results of experiments in which similarity is the variable of interest. In the context of recognition memory for sentences, the notion of perceptual similarity corresponds to the relation of semantic similarity existing between two sentences.

These arguments underlie the rationale for Experiment 1: A subject is presented with a sentence on a study-trial and asked either to form an image for it or just to comprehend it. The different encoding instructions should produce sentence representations differing in content. The imagery instructions should produce encodings that emphasize perceptual aspects of the sentence meanings, whereas the comprehension instructions should produce less elaborate, and more abstract, representations. However, whether these differences lead to differences in recognition performance depends on the nature of the distractor sentences. On a two-alternative forced-choice recognition memory test, using semantically related target and distractor sentences, the theory claims that subjects use imagery to discriminate between the sentences. This establishes the appropriate retrieval conditions for the image-derived study-trial encodings to augment recognition performance. The subjects in the comprehension condition also use imagery to dis. criminate the test sentences, but their recognition performance should not be enhanced, because the stimulus sentence encodings do not overlap sufficiently with the enriched test-trial encodings for the latter to be accurately distinguished. On the other hand, if the test sentences have a low degree of semantic similarity, it is not necessary for subjects to use imagery to discriminate between them. In this test condition, recognition accuracy should be higher than in the semantically related condi- tion, but should be relatively unaffected by the encoding instructions. This is because there is no predicted difference in the degree of overlap between the different study-trial encodings and the test-item representations. In a third test condition, corresponding to Tulving's (1981) ecphoric similarity condition, the distractor sentence is semantically similar to one of the study. trial sentences, but not to the target sentence with which it is paired. As in the dissimilarity condition, subjects do not need to use imagery to discriminate the test sentences, and thus the different encoding instructions should not produce differential performance. However, if Tulving's results can be extended to recognition memory for sentences, the overall level of recognition accuracy on this condition should be lower than on the semantically similar condition. In summary, recognition accuracy should be highest in the dissimilarity condition and lowest in the ecphoric similarity condition, and the encoding instructions should only produce differential performance on the semantic similarity condition. These predictions were tested in Experiment 1.

\section{EXPERIMENT 1}

\section{Method}

Subjects. The subjects, all volunteers, were 48 adult education students. They were run individually, and each experimental session lasted about $1 \mathrm{~h}$.

Materials. One hundred and fifty pairs of subject-verbobject $(\mathrm{S}-\mathrm{V}-\mathrm{O})$ sentences were generated such that the sentences within a pair had the same subject and object noun phrases, but different verbs. The verbs within each sentence pair were selected to be close synonyms and were matched for length $( \pm 2$ letters) and frequency of occurrence in written English (Kucera \& Francis, 1967). The set of sentence pairs was split to form two lists, Lists 1 and 2 , such that each list contained only one member of each pair. Fifteen subjects rated the sentences in List 1 for image-evoking value, and a different 15 subjects rated the sentences in List 2 . The subjects were drawn from the same pool as the experimental subjects. All the subjects were instructed to rate the sentences for ease of image formation on a scale ranging from 1 (extremely low imagery) to 7 (extremely high imagery). Each subject received a different random ordering of the sentences within a list. The correlation between the imagery values for the sentences within a pair was $\mathrm{r}=.88$, showing that the sentences were close in image-evoking value as well as in meaning.

The stimulus materials consisted of 64 sentence pairs: the 32 pairs with the highest mean imagery-evoking values and the 32 pairs with the lowest mean values. The mean imagery values for the high- and low-imagery groups were 5.47 and 2.87 , respectively. A sample of the stimulus materials, along with the corresponding distractor sentences used in the recognition tests, is given in Table 1. Forty-eight unique stimulus lists were generated; each list contained one of the sentences from each of the 64 sentence pairs. The sentences were allocated randomly to the lists, with each sentence appearing on half the lists. The order of the sentences was randomized across lists. For each sentence pair, a recognition test distractor sentence using the same subject and object noun phrases was generated. The verbs of the sentences were chosen from the same class of verbs used to generate the stimulus sentences, but did not overlap with them in meaning. In addition, they were matched for tense, length, and frequency. 
Table 1

A Sample of the Materials Used in Experiment 1, Showing Both High(H)- and Low(L)-Imagery-Value Sentences

\begin{tabular}{|c|c|c|c|c|c|}
\hline \multirow[b]{2}{*}{ Subject Noun Phrase } & \multirow{2}{*}{$\begin{array}{c}\text { Imagery } \\
\text { Value }\end{array}$} & \multicolumn{3}{|c|}{ Verbs } & \multirow[b]{2}{*}{ Object Noun Phrase } \\
\hline & & & & Distractor & \\
\hline The man & $\mathrm{H}$ & strolled & sauntered & cycled & through the park \\
\hline The prisoner & $\mathbf{H}$ & scribbled & scrawled & sealed & his final letter \\
\hline The attendant & $\mathrm{H}$ & drenched & watered & planted & the flower beds \\
\hline The schoolboy & $\mathrm{L}$ & invented & concocted & recited & the heroic stories \\
\hline The politicians & $\mathrm{L}$ & governed & managed & exploited & the poor colonies \\
\hline The creatures & $\mathrm{L}$ & depleted & exhausted & covered & their food supply \\
\hline
\end{tabular}

Three types of test item were constructed to be used in a two-alternative forced-choice recognition memory test. The three types corresponded to the three experimental test conditions, as follows: Using a notation based on Tulving (1981), let $\mathrm{S} 1, \mathrm{~S} 2, \mathrm{S3}, \ldots$ designate the stimulus sentences presented in the study phase, $\mathrm{S}^{\prime}, \mathrm{S} 2^{\prime}, \mathrm{S} 3^{\prime}, \ldots$ the sentences that are closely related to them in meaning, and $\mathrm{D} 1^{\prime}, \mathrm{D} 2^{\prime}, \mathrm{D} 3^{\prime}, \ldots$ the corresponding distractor sentences. The three experimental test conditions and associated test items can now be defined as: (1) synonym condition ( $\mathrm{S} 1-\mathrm{S} 1^{\prime}$ ), in which the two alternatives in a test item are closely related in meaning; (2) distractor condition (S1-D1'), in which the alternative sentences only share the same subject and object noun phrases; and (3) ecphoric similarity condition (S1-S2'), in which the two sentences are not synonymous with each other but the nontarget sentence is synonymous with another previously studied sentence (S2). This last condition was included to test Tulving's (1981) proposal concerning different types of similarity and their effects on recognition performance. In the two-alternative, forcedchoice recognition test, each subject was tested on 48 items, 16 items in each of the three test conditions. Each test item presented the subject with one of the stimulus sentences and a "lure." Since the stimulus sentences associated with the lures in the $\mathbf{S 1 - S 2}$ ' condition did not appear in the recognition test, the 64 stimulus sentences were tested using only 48 pairs of alternatives. For each stimulus list, a unique recognition memory test list was constructed such that the target sentences appeared in the same ordinal position on both lists. This procedure attempted to balance the item lag between study and test on any target sentence. On each test, half the items in each condition presented pairs of high-imagery sentences, and the other half of the items consisted of low-imagery sentences. Each stimulus sentence served equally often in the three test conditions and as one of the sentences used to derive the lures in the S1-S2' condition. Furthermore, in this latter condition, the target sentences and lures were matched for imagery; that is, the 8 high-imagery stimulus sentences were paired with the 8 highimagery sentence lures, and similarly for the low-imagery sentences.

Design. The 48 subjects were assigned randomly to receive one of the stimulus lists in one of the two study conditions, the comprehension condition or the imagery condition. Equal numbers of subjects were assigned to the two conditions. Fol lowing this, all the subjects were asked to complete the twoalternative forced-choice recognition memory test corresponding to the stimulus list that they had received. This was a 2 (study condition: comprehension vs. imagery) $\times 3$ (test condition: S1-S1' vs. S1-D1' vs. S1-S2') x 2 (imagery-evoking value: high vs. low) design, with the first variable being between subjects and the last two being within subjects.

Procedure. The two study conditions were identical except for the instructions given to the subjects. All the stimulus sentences were presented in the auditory modality over headphones connected to a tape recorder controlled by a computer. The stimulus lists were presented in the auditory mode in an attempt to minimize any modality interference effects between the two study conditions (Eddy \& Glass, 1981). Each trial began with a 1-sec auditory signal that served as a "ready" signal. This was followed by a stimulus sentence. A digital timer was started by a $1000-\mathrm{Hz}$ pulse on a separate channel of the tape recorder. The pulse was set such that the timer was started at the onset of the last syllable of each sentence. The subjects responded by pressing a button placed under the first finger of their dominant hand. To facilitate rapid responding, the subjects rested their fingers on the button between responses. The response times were recorded. There was a 5 -sec interval between the subjects' responses and the start of the next trial.

In the comprehension condition, the subjects were instructed to listen to the stimulus sentence and to press the response button as soon as they felt they had fully comprehended the sentence. They were informed that their memory for the sentences would be tested afterwards.

In the imagery condition, the subjects were asked to generate a mental image that depicted the meaning of the stimulus sentence and to press the response button as soon as they had formed a complete image. These subjects were also informed that their memory for the sentences would be tested afterwards.

Each subject received 10 practice trials on sentences similar in nature to the stimulus sentences. After the experimenter answered questions clarifying any uncertainties regarding the instructions, the stimulus sentences were presented.

Following presentation of the stimulus sentences, the subjects were given an interpolated task for $2 \mathrm{~min}$. The subjects were required to write down the names of as many English cities as they could think of. The subjects were then asked to complete the two-alternative forced-choice recognition test appropriate to the stimulus list they had received. The test lists were presented with a projection tachistoscope; $10 \mathrm{sec}$ were allowed for each test item. The subjects were asked to turn to a page of the response booklet on which were listed the numbers 1 through 48 . The letters " $a, b$ " were printed next to each number; these identified the alternative sentences in each item. On the same line were printed the digits " 1, ," 2 ," and " 3 ," which were to be used for the confidence-rating judgments. The subjects were told that on each test item they would see two sentences, labeled " $a$ " and " $b$," and that one, and only one, of the sentences had been presented as a stimulus sentence. They were asked to indicate by a check mark against the appropriate letter which sentence of the two they had heard during the first part of the experiment. It was emphasized that the subjects should mark one sentence for each test item, and should guess if they were unsure. The subjects also gave a confidence judgment to accompany their decisions by circling one of the digits on the 3 -point scale, with 3 designating high confidence and 1 representing guessing.

\section{Results}

A preliminary analysis of the subject's recognition performance showed that the data could be pooled over the entire set of stimulus sentences; that is, performance was symmetrical for the synonymous sentences within 
Table 2

Hit Rates and Mean Confidence Ratings for Hits in the Three Conditions on the Forced-Choice Recognition Test, for the Two Study Conditions and Sentence Types Used in Experiment 1

\begin{tabular}{|c|c|c|c|c|c|c|c|}
\hline \multirow[b]{3}{*}{ Study Condition } & \multirow{3}{*}{$\begin{array}{l}\text { Imagery } \\
\text { Value }\end{array}$} & \multicolumn{6}{|c|}{ Test Conditions } \\
\hline & & \multicolumn{3}{|c|}{ Hit Rates } & \multicolumn{3}{|c|}{ Mean Confidence Ratings } \\
\hline & & S1-S1' & S1-D1' & S1-S2' & S1-S1' & S1-D1' & S1-S2' \\
\hline \multirow{2}{*}{ Comprehension } & High & .67 & .82 & .64 & 2.11 & 2.27 & 2.07 \\
\hline & Low & .69 & .81 & .65 & 2.04 & 2.23 & 1.91 \\
\hline \multirow{2}{*}{ Image Generation } & High & .81 & .81 & .63 & 2.25 & 2.32 & 2.09 \\
\hline & Low & .77 & .79 & .66 & 2.19 & 2.28 & 2.01 \\
\hline
\end{tabular}

a pair. Table 2 shows the hit rates and the mean confidence ratings for hits in the three conditions on the forced-choice recogntion test, for each study condition and sentence type.

A 2 (study condition) $\times 3$ (test condition) $\times 2$ (imagery value) mixed analysis of variance was performed on the hit rates. The results of the analysis showed that two main effects were significant: Image generation leads to better recognition performance than does comprehension $[F(1,46)=5.14, \mathrm{p}<.05]$, and performance is affected by the nature of the lures $[F(2,92)=7.28$, $\mathrm{p}<.01$ ]. In addition, there was a reliable interaction between study condition and recognition test condition $[F(2,92)=6.83, p<.01]$. The results of a NewmanKeuls test of pairwise comparisons indicated that recognition performance was significantly better in Condition S1-D1' than in the other two test conditions; the differences in performance on these latter two conditions were nonsignificant. However, this result is true only for performance in the comprehension study condition. The data show that image-generation instructions led to improved recognition performance in Condition S1-S1' than in Condition S1-S2'. Furthermore, these results seem to be consistent for both highand low-imagery sentences. The means show no systematic differences in recognition performance as a function of imagery-evoking value of the stimulus sentences $(\mathrm{F}<2.50)$.

The means of the confidence judgments for hits are shown in Table 2 as a function of study and test conditions and sentence type. These means reflect the same general effects reported for correct responses. An analysis of variance carried out on the confidence judgment data showed that the study- and test-condition main effects were again significant $[F(1,46)=4.25, p<.05$, and $F(2,92)=4.76, p<.025$, respectively $]$, as was the study $x$ test condition interaction $[F(2,92)=4.02$, $\mathrm{p}<.05]$. A number of studies have reported strong positive correlations between recognition accuracy on forced-choice tests and subjects' confidence in their judgments (Bower \& Glass, 1976; Tulving, 1981).

\section{Discussion}

The findings confirm the prediction that imageryencoding instructions produce more sensitive recognition memory for sentences than do comprehension instructions, but only in the semantic similarity (S1-S1') test condition. In the other two test conditions, encoding instructions did not differentially affect recognition accuracy. However, the fact that there were no reliable effects associated with image-evoking value is rather disturbing, given the theoretical rationale of the experiment. Accounting for the data in terms of overlapping memory traces produced through image processing at encoding and retrieval would lead one to predict that the quality of that processing for different sentences, presumably reflected in the image-evoking values, should influence recognition performance. One plausible explanation for the noneffect relates to the content of the experimental sentences. On inspection of the sentences, it is apparent that, whereas the verbs range from high to low in terms of image-evoking value, the subject and object noun phrases represent a more restricted set, tending to be of high imagery. Thus, it is possible that the materials span a relatively restricted range of imageevoking values. Subjects may use imagery to discriminate the test sentences providing that the subject and object concepts are relatively easy to image, irrespective of the image-evoking value of the verbs. By using a wider range of sentences, including those with abstract subject and object concepts, it should be possible to test whether the noneffect due to image-evoking value is a product of the sentence sample used or a general finding that would be detrimental to the theory being proposed. Experiment 2 repeated Experiment 1, using such sentences.

\section{EXPERIMENT 2}

\section{Method}

Materials. Fifty additional pairs of $\mathrm{S}-\mathrm{O}-\mathrm{V}$ sentences similar to those used in Experiment 1 were generated. The sentences within each pair shared the same subject and object noun phrases, but contained different verbs that had been selected as close synonyms. However, unlike the pairs of sentences used in Experiment 1, the shared subject nouns were chosen from a set of low-imagery words (imagery value less than 3.5 on a scale range of $1-7$ in the Toglia \& Battig, 1978, norms). These new sentence pairs were added to the set of stimulus sentence pairs used in Experiment 1 to form a total set of 114 sentence pairs that were rated for imagery-evoking value and comprehensibility. Two lists of sentences were produced, Lists 1 and 2 , such that each list contained only one member of each sentence pair. 
In all, 56 subjects rated the sentence pairs, using the procedure described previously. For each list, half the subjects were instructed to rate the sentences for ease of image formation, and half for ease of comprehension.

As had been found in generating the materials for Fxperiment 1 , the imagery-evoking values of the sentences within a pair correlated highly $(r=.85)$, as did the comprehensibility ratings $(r=.88)$. Furthermore, the imagery and comprehensibility ratings correlated quite highly with each other $(r=.48)$, showing that high-imagery sentences tended to be rated as easier to understand than low-imagery sentences. More importantly, the original 64 stimulus sentences were rated higher in imageevoking value (mean $=5.12$ ) than were the new sentences containing low-imagery subject nouns (mean $=3.05)[\mathrm{t}(27)=3.88$, $\mathrm{p}<.001]$. The new rating data also validated the ordinal properties of the original imagery ratings; the rank order correlation between the two sets of imagery ratings for the original stimulus sentences was $r=.86$.

Sixty-four sentence pairs were chosen to be used as the stimuli in Experiment 2. The 32 sentences with the lowest mean imagery-evoking values were used, all of which had low-imagery subject nouns. The 32 high-imagery sentence pairs were chosen from the set of 60 sentence pairs with the highest mean imagery ratings. Although high- and low-imagery sentences appeared to differ systematically in terms of mean comprehensibility rating, there was sufficient variance in the set of 60 sentence pairs to match the high- and low-imagery sentences for comprehensibility. Each high-imagery sentence pair was matched with a lowimagery sentence pair for sentence length (tolerance, \pm 1 syllable) and comprehensibility (tolerance, \pm .3 rating points). The mean imagery-evoking values for the high- and low-imagery sentence pairs were 4.88 and 2.85 , respectively, and the equivalent mean comprehensibility ratings were 3.61 and 3.47 , respectively.

A recognition test distractor sentence was generated for each sentence pair, using the same subject and object noun phrases but semantically different verbs. A sample of the stimulus and test sentences is given in Table 3. Forty-eight stimulus lists and associated recognition test lists were constructed in the same way as were those in Experiment 1.

Subjects. Forty-eight new subjects were drawn from the same population used for Experiment 1. All the subjects were tested individually; each experimental session lasted about $1.25 \mathrm{~h}$.

Design and Procedure. The general procedures and design of Experiment 2 were the same as those employed in Experiment 1 . The subjects were assigned randomly to receive one of the stimulus lists in one of two study conditions, comprehension or image generation, and then completed the appropriate twoalternative recognition test comprising items in three test conditions, S1-S1', S1-D1', and S1-S2'.

\section{Results}

Looking first at the analysis of the response times for the two study conditions and sentence types, we find that both main effects were reliable, as was the study condition $x$ sentence type interaction $[F(1,46)=13.72$, $\mathrm{p}<.001]$. In general, it takes longer to construct an image for a sentence (mean response time $=1,654 \mathrm{msec}$ ) than it does to comprehend it (mean response time $=$ $1,228 \mathrm{msec})[F(1,46)=22.61, \mathrm{p}<.001]$. Furthermore, the response times for high-imagery sentences (mean response time $=1,322 \mathrm{msec}$ ) are shorter than the response times for low-imagery sentences (mean response time $=1,561 \mathrm{msec})[\mathrm{F}(1,46)=8.11, \mathrm{p}<.01]$. A pairwise comparison of the mean response times showed that the high- and low-imagery sentences were comprehended equally quickly (difference in mean response times was $22 \mathrm{msec}$ ), but it took much longer to form images for low-imagery sentences (mean response time $=$ $1,882 \mathrm{msec}$ ) than for high-imagery sentences (mean response time $=1,426 \mathrm{msec}$ ). This finding validates the design in showing that the stimulus sentences are distinguishable in terms of imagery properties but not in terms of ease of understanding.

The analysis of the subjects' recognition performance confirmed the findings of the previous experiment; both the study and test conditions produced significant effects $[F(1,46)=4.68, p<.05$, and $F(2,92)=8.47$, $\mathrm{p}<.001$, respectively , and the study $\mathrm{x}$ test condition interaction was also reliable $[\mathrm{F}(2,92)=7.64, \mathrm{p}<.001]$. However, in this experiment, there was also a reliable main effect due to imagery-evoking value $[F(1,46)=$ $5.07, \mathrm{p}<.05]$, and the triple interaction was significant $[F(2,92)=6.82, p<.01]$. The hit rates in the three test conditions, for each study condition and sentence type, are shown in Table 4. Individual paired comparisons contrasting test conditions within study conditions were performed. The analysis showed that, for highimagery sentences, recognition performance was better in the S1-D1' condition than in the other two test conditions, irrespective of the encoding instructions, with the exception that performance in the $\mathrm{S} 1-\mathrm{S} 1^{\prime}$ condition for subjects receiving imagery instructions was as good as in Condition S1-D1'. However, the pattern of results differed for low-imagery sentences in that the latter exception did not hold; performance in Condition S1-D 1' was always better than in the other test conditions, regardless of study condition. Furthermore, Test Conditions S1-S1' and S1-S2' could not be differentiated on the basis of recognition performance.

Table 3

A Sample of the Materials Used in Experiment 2, Showing Both High(H)- and Low(L)-Imagery-Value Sentences

\begin{tabular}{cccccc}
\hline & Imagery & & \multicolumn{2}{c}{ Verbs } & \\
\cline { 3 - 5 } Subject Noun Phrase & Value & & Synonyms & Distractor & Object Noun Phrase \\
\hline The builders & H & twisted & warped & erected & the support frame \\
The detective & H & searched & scoured & tidied & his dingy office \\
The gangster & H & disliked & resented & ignored & the biased questions \\
The statistics & L & enhanced & enriched & supported & the report's findings \\
The scheme & L & required & demanded & received & total cooperation \\
The rumors & L & ridiculed & derided & reinforced & the man's reputation \\
\hline
\end{tabular}


Table 4

Hit Rates in the Three Conditions on the Forced-Choice Recognition Test, for the Two Study Conditions and Sentence Types Used in Experiment 2

\begin{tabular}{lcccc}
\hline & & \multicolumn{3}{c}{ Test Conditions } \\
\cline { 3 - 5 } Study Condition & Value & S1-S1' & S1-D1' & S1-S2' \\
\hline \multirow{2}{*}{ Comprehension } & High & .70 & .84 & .66 \\
& Low & .67 & .82 & .65 \\
Image Generation & High & .82 & .83 & .69 \\
& Low & .71 & .80 & .64 \\
\hline
\end{tabular}

The analysis of the subjects' confidence ratings totally supported the pattern of the recognition accuracy data.

\section{Discussion}

The findings of Experiment 2 indicate that the lack of effects due to image-evoking value in Experiment 1 were related to the restricted range of stimulus sentences used. The rating data clearly demonstrate that the original sentences were drawn from a more restricted set, on the basis of image-evoking value, than were the present materials. However, the original findings still stand, but with an additionai constraint. Recognition accuracy is enhanced in the S1-S1' test condition for subjects performing the image-generation task, but as Experiment 2 showed, this result only holds for highimagery sentences. For low-imagery sentences containing abstract noun concepts, recognition accuracy is at the same low level on both the S1-S1' and S1-S2' conditions, regardless of the encoding task. Thus, it seems that the quality of a mental image, or the ease with which one can be derived for a given sentence, influences either the choice or the effectiveness of the retrieval strategy in discriminating between semantically similar sentences. Furthermore, the results of the two experiments seem to imply that the image-evoking quality of the noun concepts, rather than the verb concept, is the major determining factor in the subjects' use of imagery.

An alternative explanation of the data is that subjects elaborate the test sentences in the S1-S1' condition by using purely semantic processing rather than imagery (Kieras, 1978). The rationale of the experiments remains essentially unchanged, except that it is unnecessary to use imagery to discriminate the test alternatives; semantic elaboration of the sentence representations is sufficient. Moreover, the image-evoking-value effects can be explained in terms of the argument that high-imagery sentences produce richer semantic encodings than do low-imagery sentences, leading to increased studyand test-trial encoding overlap. Experiment 3 was designed to counter this explanation.

In the study phase of Experiment 3, all the subjects engaged in a sentence classification task in which they were required to make a binary response on the basis of one of three criteria. The criteria differed in terms of both the type and depth of processing required to make a judgment. In one condition, the subjects were asked to classify the sentences according to how EASY or HARD they were to understand. In a second condition, the classification criterion was the ease with which a sentence evoked a mental image. Finally, in a third condition, referred to as the "implied actors" condition, the subjects had to classify the sentences on the basis of whether they thought a sentence directly implied the involvement of actors not explicitly mentioned. For example, the sentence "The scheme required total cooperation" is likely to produce a positive response because the concept "cooperation" alludes to implicit actors who are required to cooperate for the scheme to be successful. On the other hand, additional actors cannot be inferred from the sentence "The detective searched his dingy office," because none of the component concepts requires the involvement of actors in addition to the detective. In all other respects, Experiment 3 was identical to Experiment 2.

The rationale underlying the use of the three encoding tasks hinges on the assumption that the "implied actors" classification task involves deeper semantic processing than does the comprehension classification task. In the former task, a subject is required to draw specific inferences based on the conceptual content of a sentence, in addition to constructing a representation for it. Most current theories of sentence comprehension (Norman \& Rumelhart, 1975; Schank, 1975) propose that such inferences require the representation of the verb to be expanded through decomposition in to more primitive semantic elements, producing a semantically elaborated trace. There is considerable evidence that sentence classification tasks of this type produce more elaborate encodings for sentences than do simple comprehension tasks (Jacoby \& Craik, 1979; Kolers, 1975). Thus, the study-trial encodings resulting from the "implied actors" task should incorporate expanded verb representations, in comparison with those produced by the comprehension task. The $\mathrm{S} 1-\mathrm{S} 1^{\prime}$ test sentences differ only in terms of their verb components, so if they are discriminated through purely semantic processing, then increased recognition accuracy for subjects performing the "implied actors" task should result. This is because both the study- and test-trial encodings will contain expanded representations for the verbs, which increases the degree of overlap between the encodings for a target item. However, if subjects use imagery to elaborate the S1-S1' test-trial encodings, the additional perceptual information they incorporate will be less compatible with the sentence representations produced by the "implied actors" task, but will match up with the perceptually enriched encodings produced by the imagery classification task. This should result in a higher degree of overlap between target sentence encodings associated with the latter task, which should produce more sensitive discrimination between the test alternatives. Thus, S1-S1' recognition accuracy should be highest for sub- 
jects performing the imagery classification task, but only for high-imagery sentences. There may also be significant differences in performance between the other two study conditions, but such differences should not be limited to the data for high-imagery sentences in the S1-S1' test condition.

\section{EXPERIMENT 3}

\section{Method}

Subjects. Seventy-two subjects were drawn from the same population used for Experiment 1 .

Materials. The experimental materials comprised 72 stimulus sentence lists and corresponding forced-choice recognition test lists, generated using the 64 stimulus sentence pairs and distractor sentences that had been used in Experiment 2. Of the 32 low-imagery sentences, the experimenter classified 18 as positive and 14 as negative examples of sentences that imply the involvement of actors not explicitly mentioned. For the high-imagery sentences, however, the corresponding figures were 21 and 11 . To balance the ratio of responses for the two types of stimulus sentence, three new triples were substituted in the high-imagery set, so that the ratio of probable responses was $18: 14$ for both sets. The new sentence triples were chosen from the original pool of stimulus sentences generated for Experiment 2, and matched the substituted sentences on image-evoking value and comprehension rating.

Design. The experimental design was similar to that used in Experiment 2, except that it was extended to include a third study condition. The subjects were assigned randomly to receive one of the stimulus lists in one of the three study conditions, the "comprehension" condition, the "imagery" condition, or the "implied actors" condition.

Procedure. All three study conditions involved a binary classification task, but differed in terms of the basis on which the judgment had to be made. Again, all the sentences were presented in the auditory modality. The response keyboard had two microswitches on which the subject rested one finger from each of his or her right and left hands. The response keys were used to terminate the timer. The subject's responses and response times were recorded on the computer.

In the "comprehension" condition, the subjects were asked to listen to each stimulus sentence, to decide whether they would rate it as EASY or HARD to understand, and to make the appropriate response as quickly as possible. The subjects were given lengthy instructions concerning how they should interpret the response categories.

The subjects in the "imagery" condition were given similar instructions, except that they were asked to rate the sentences as either EASY or HARD on the basis of the ease with which a sentence evoked a mental image.

The "implied actors" condition was slightly different in that the subjects were asked to make a YES/NO response for each sentence on the basis of whether it implied the involvement of actors other than those explicitly mentioned. The subjects were given extensive instructions about what constituted a direct implication of additional actors. As in the other two study conditions, emphasis was placed on both speed and accuracy of response.

All the subjects were told that their memory for the sentences would be tested at the end of the experiment. Each subject received 20 practice trials on sentences similar to those used in the actual experiment. The mapping of response categories onto response keys was alternated between subjects. At the end of the experiment, each subject was given the appropriate forced-choice recognition memory test to complete, the procedure being identical to that used in Experiment 1.

\section{Results}

The concordance between the experimenter and subject classifications in the "implied actors" condition was .76 , showing that the subjects were completing the task as specified. In the analysis of the response times, all the responses were included. Again, both main effects were reliable. In general, high-imagery sentences (mean response time $=1,662 \mathrm{msec}$ ) produced faster responses than did low-imagery sentences (mean response time $=$ $1,771 \mathrm{msec})[\mathrm{F}(1,69)=5.87, \mathrm{p}<.05]$, and response time was influenced by the nature of the classification task $[F(2,69)=6.85, p<.01]$. The interaction was also significant $[\mathrm{F}(2,69)=6.32, \mathrm{p}<.01]$. A comparison-ofmeans test showed that response times were faster on the comprehension classification task $(1,542 \mathrm{msec})$ than on the other two tasks (imagery task, $1,773 \mathrm{msec}$; "implied actors" task, $1,835 \mathrm{msec})$. These last two conditions could not be separated. Moreover, only on the imagery condition did the two types of stimulus sentence produce reliable differences in response time (difference in means $=192 \mathrm{msec}$ ).

A mixed analysis of variance of the subjects' recognition performance showed that all three main effects were significant. That is, performance was influenced by the study task $[\mathrm{F}(2,69)=3.87, \mathrm{p}<.05]$, test condition $[F(2,69)=7.86, p<.001]$, and sentence type $[F(1,69)=4.26, p<.05]$. The study $x$ test condition interaction was significant $[F(4,138)=3.16, p<.05]$, as was the three-way interaction $[F(4,138)=4.87$, $\mathrm{p}<.01]$. A comparison-of-means test showed that recognition accuracy was equally high in the "imagery" and "implied actors" conditions in comparison with the level of performance on the "comprehension" condition. The differences in performance on the test conditions were in the same direction as had been found in the previous two experiments, as shown in Table 5. Individual paired comparisons contrasting test conditions within study conditions revealed additional structure. Performance on the S1-D1' test condition was not affected by study condition or sentence type. Performance on the other two test conditions was at an equally low level, independent of study condition and sentence type, but there were two important exceptions to this. First, as had been found in Experiment 2, recognition ac-

Table 5

Hit Rates on the Three Test Conditions in Experiment 3 as a Function of Encoding Task and Sentence Type

\begin{tabular}{lccccc}
\hline \multirow{2}{*}{$\begin{array}{c}\text { Encoding Classifi- } \\
\text { cation Task }\end{array}$} & Mean & $\begin{array}{c}\text { Imagery } \\
\text { Value }\end{array}$ & \multicolumn{3}{c}{ Test Condition } \\
\cline { 4 - 6 } \cline { 4 - 5 } Comprehension & .73 & High & .70 & .84 & .68 \\
& & Low & .69 & .80 & .65 \\
Implied Actor & .77 & High & .69 & .85 & .75 \\
& & Low & .75 & .82 & .73 \\
Imagery & .76 & High & .85 & .84 & .68 \\
Mean & & Low & .71 & .82 & .66 \\
\cline { 5 - 6 } & & & .73 & .83 & .69 \\
\hline
\end{tabular}


curacy on the S1-S1' condition was enhanced to the level of S1-D1' performance for high-imagery sentences in the imagery study condition. Second, on the S1-S2' test condition, recognition accuracy was higher in the "implied actors" condition, irrespective of sentence type, than in the other two study conditions, but did not reach the level of S1-D1' performance. The pattern of the recognition accuracy data was, as in the other two experiments, reflected in the subjects' confidence judgments.

\section{Discussion}

The pattern of the data in the comprehension and imagery conditions was unchanged from that in Experiment 2 . The only difference between the two sets of data relates to the response times on the imageryrelated study tasks. The convergence of response times for high- and low-imagery sentences in the classification task is to be expected, given the nature of the task. The very high- and very low-imagery sentences are relatively easy to classify and produce low response times, bringing the means for the two types of sentences together.

Inclusion of the data for the "implied actors" condition brings out more interesting comparisons. The high level of recognition performance in this condition, relative to that in the comprehension condition, is in line with the majority of findings in the levels-of-processing literature (Cermak \& Craik, 1979). Also, the response time data show that the task is relatively unaffected by the imagery variable, implying that it involves purely semantic processing. Moreover, the differences in performance in the S1-S1' test condition for subjects in the "implied actors" and "imagery" conditions lead one to the conclusion that the semantically elaborated encodings produced by the former task have a relatively low degree of overlap with the test sentence encodings. Only imagery-related encoding tasks seem to produce representations that overlap sufficiently with the differentiated S1-S1' test-trial encodings to augment retrieval. This result strongly suggests that these latter encodings are the product of image-related processing, thereby confirming the original idea that imagery is used to discriminate semantically similar sentences, albeit only when the concreteness of the content nouns makes it a feasible strategy.

Two interesting aspects of the data relate to the differences in recognition performance in the "implied actors" condition. First, there is an enhancement in the accuracy in the S1-S2' condition. Second, performance in the S1-S1' condition is better for low-imagery sentences than for high-imagery sentences, although this difference did not quite reach significance. Both findings might be explained by the notion that the subjects take advantage of the semantically elaborated study-trial encodings to improve their recognition accuracy. In the S1-S2' test condition, subjects may adopt the strategy of producing semantically rich representations for test sentences, leading to an increased overlap in the studyand test-trial encodings for target items. This strategy is ineffective for subjects in the other study conditions because the study-trial encodings either are too impoverished or are incompatible, depending on the encoding task. The "implied actors" subjects may also use this strategy in discriminating the low-imagery test sentences in the S1-S1' condition as an alternative to using imagery that is inhibited by the abstract content of the sentences. Again, the resulting increase in the overlap of the target item encodings produces more sensitive performance. However, the same subjects use imagery to discriminate the high-imagery sentences in the $\mathrm{S} 1-\mathrm{S} 1^{\prime}$ condition, producing encodings that are less compatible with the traces laid down during the study phase, which leads to poorer performance.

\section{GENERAL DISCUSSION}

The three experiments showed that on a twoalternative forced-choice recognition memory task using semantically similar distractors, recognition accuracy for high-imagery target sentences is enhanced if subjects perform imagery-related tasks on the study trials. Under the same test conditions, comprehension and other related semantic encoding tasks do not produce equivalent improvements in performance. Furthermore, the first two experiments show that the high-imagery constraint excludes only those sentences that contain abstract subject and object nouns; the effect seems to be independent of the image-evoking qualities of the verb component.

It could be argued that the differences in recognition performance are an artifact of the differences in the study trial durations; on average, the subjects processed the sentences considerably longer on the image-related tasks than on the semantic tasks. However, this explanation does not account for the within-condition differences in recognition accuracy. For example, it would predict an imagery effect on performance that is in the opposite direction from the one found. The imagegeneration times for high-imagery sentences are significantly shorter than those for low-imagery sentences, but recognition accuracy is lower for the latter than for the former, particularly on the semantic similarity condition. The general findings seem to be consistent across the three experiments, regardless of the encoding tasks employed.

The present results strongly suggest that the improved performance in the S1-S1' test condition for subjects in the imagery-encoding conditions can be attributed to the generation of images as part of the processing required to differentiate the test sentences. This conclusion is concordant with the results of Fisher and Craik (1977, 1980), who showed that elaborate encodings produced by study tasks are effective in enhancing later recognition only if the encoding environment is reinstated at the time of retrieval. That is, the encodings produced by the imagery tasks are effective in augmenting performance because imagery is also used in differentiating the S1-S1' test sentences. Moreover, using different encoding 
and testing environments in a recognition memory task, Groninger and Groninger (1982) showed that similar images of the same stimulus-item referent have to be generated at encoding and retrieval for image congruence to enhance accuracy.

Kieras (1978) argued, rightly, that some form of semantic interpretation, however minimal, needs to be established before image processing can begin. Given that this is true, why should subjects evoke imagery to discriminate the test sentences rather than continue processing them semantically? One simple reason might be that the high-imagery sentences evoke it. That is, imagery is the most readily available processing medium. However, this explanation is not supported by the present data. The first two experiments showed that subjects used imagery to differentiate test sentences containing verbs spanning a range of image-evoking values. The S1-S1' alternatives differ only in terms of their verb components, so it seems that subjects use image processing regardless of the imageability of the major elements to be discriminated. Only when the noun concepts are difficult to image does the strategy break down. An alternative reason for using imagery might be that it provides the most direct access to information that can be used to differentiate the sentences. It may be easier to elaborate the verb components through the use of imagery than to expand their representations through purely semantic processing. Taking this argument further, it is possible that the semantic system does not contain the information necessary for maximizing the discrimination of the sentences. This information may be represented only in perceptual codes accessed through imagery. This possibility is partially supported by the work of Walter and Fox (1981). They argued that, in sentence memory, one of the primary objectives of semantic processing is to construct encoding configurations that provide easy access to perceptual information. Thus, it is possible that subjects use imagery to make available the perceptual information that maximizes the differentiation of the test sentence encodings.

Finally, it seems, on first inspection, that the present data support Tulving's (1981) findings in that overall recognition performance is highest in the S1-D1' test condition, next highest in Condition S1-S1', and worst in Condition S1-S2'. Thus, Tulving's results, which were based on forced-choice tasks involving perceptual similarity, seem to generalize to tasks involving semantic similarity, and in particular to recognition memory for sentences. Unfortunately, the picture becomes cloudy once you isolate the data for the different study-trial tasks. From the data in Table 5, for example, it is apparent that performance is equally poor on Conditions $\mathbf{S} 1-S 1^{\prime}$ and $\mathbf{S} 1-S 2^{\prime}$ for all study conditions and sentence types, except for high-imagery sentences in the imagery study condition. In this latter case, Conditions S1-S1' and S1-D1' are indistinguishable in terms of level of accuracy. However, this parity in performance is not particularly significant, because S1-D1' perfor- mance is highly dependent on the nature of the distractor sentences employed and their precise relationship to the target sentences. Tulving's results, then, hold only for the specific case of high-imagery sentences under imagery-encoding instructions. On the basis of the assumption that imagery involves perception-like processes, a supposition for which there is considerable evidence (Finke, 1980), it might be concluded that the differences in recognition accuracy shown in the present data reflect the same set of processes that underlie Tulving's original findings. This idea is consistent with Tulving's conjecture that his reversal of the classical test-item similarity effect is possibly due to subjects' adopting the strategy of elaborative processing of the retrieval information in the S1-S1' condition. This speculation leads one to conclude either that Tulving's findings hold only for perceptually related test items or that the degree of semantic similarity used in the S1-S1' condition is not comparable to the degree of perceptual similarity used by Tulving. However, the boundary limits of these results require greater empirical specification before such speculations can be taken further.

\section{REFERENCE NOTE}

1. Anderson, J. R. Recognition confusions in sentence memory. Unpublished manuscript, Stanford University, 1972.

\section{REFERENCES}

ANderson, J. R., \& Bower, G. H. Human associative memory. Washington, D.C: Winston, 1973.

BegG, I., \& Paivio, A. Concreteness and imagery in sentence meaning. Journal of Verbal Learning and Verbal Behavior, $1969,8,821-827$

Bowen, G. H., \& Glass, A. L. Structural units and the redintegrative power of picture fragments. Journal of Experimental Psychology: Human Learning and Memory, 1976, 2, 456-466.

Cermak, L. S., \& Craik, F. I. M. (EDs.). Levels of processing in human memory. Hillsdale, N.J: Erlbaum, 1979.

EDDY, J. K., \& GLAss, A. L. Reading and listening to high and low imagery sentences. Journal of Verbal Learning and Verbal Behavior, 1981, 20, 333-345.

Finke, R. A. Levels of equivalence in imagery and perception. Psychological Review, 1980, 87, 113-132.

Fisher, R. P., \& Craik, F. I. M. Interaction between encoding and retrieval operations in cued recall. Journal of Experimental Psychology: Human Learning and Memory, 1977, 3, 701-711.

Fisher, R. P., \& Craik, F. I. M. The effects of elaboration on recognition memory. Memory \& Cognition, 1980, 8, 400-404.

Glass, A. L., Eddy, J. K., \& Schwanenfluael, P. J. The verification of high and low imagery sentences. Journal of Experimental Psychology: Human Learning and Memory, 1980, 6, 692-704.

Groninger, L. D., \& Groninger, L. K. Function of images in the encoding-retrieval process. Journal of Experimental Psychology: Learning, Memory and Cognition, 1982, 8, 353-358.

JACOBY, L. L., \& Craik, F. 1. M. Effects of elaboration on encoding and retrieval: Trace distinctiveness and recovery of initial context. In L. S. Cermak \& F. I. M. Craik (Eds.), Levels of processing in human memory. Hillsdale, N.J: Erlbaum, 1979.

KIERAs, D. Beyond pictures and words: Alternative information processing models for imagery effects in verbal memory. Psychological Bulletin, 1978, 85, 532-554.

Kole rs, P. A. Memorial consequence of automatized encoding. 
Journal of Experimental Psychology: Human Learning and Memory, 1975, 1, 689-701.

Kosslyn, S. M. Image and mind. Cambridge, Mass: Harvard University Press, 1980.

Kučera, H., \& Francis, W. N. Computational analysis of present-day American English. Providence, R. I: Brown University Press, 1967.

NAGAE, S. Nature of discriminating and categorizing functions of verbal labels on recognition memory for shape. Journal of Experimental Psychology: Human Learning and Memory, 1980, 6, 421-429.

Norman, D. A., \& Rumelhart, D. E. Explorations in cognition. San Francisco: Freeman, 1975.

Paivio, A. Imagery and verbal processes. New York: Holt, Rinehart \& Winston, 1971.

Schank, R. C. The role of memory in language processing. In C. N. Cofer (Ed.), The structure of human memory. San Francisco: Freeman, 1975.
Toglin, M. P., \& BAtтig, W. F. Handbook of semantic word norms. Hillsdale, N. J: Erlbaum, 1978.

TutviNG, E. Similarity relations in recognition. Journal of Verbal Learning and Verbal Behavior, 1981, 20, 479-496.

Tulving, E., \& Thompson, D. M. Encoding specificity and retrieval processes in episodic memory. Psychological Review, 1973, 80, 352-373.

Walten, D. A., \& Fox, D. The role of perceptual and semantic elaboration in high- and low-imagery sentence recall. Memory \& Cognition, 1981, 9, 625-630.

Weaver, G. E., \& Stanny, C. T. Short-term retention of pictorial stimuli as assessed by a probe recognition technique. Journal of Experimental Psychology: Human Learning and Memory, 1978, 4, 55-65.

(Manuscript received December 10, 1982; revision accepted for publication July 29,1983 .) 